\title{
Beyond fetal magnetic resonance diagnosis of corpus callosum agenesis
}

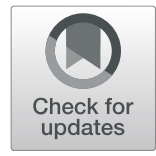

Rania S. M. Ibrahim ${ }^{1,2^{*}}$ and S. Emad-Eldin ${ }^{3}$

\begin{abstract}
Background: Corpus callosum agenesis (CCA) is a midline congenital anomaly. Routine prenatal 2D ultrasonography (2D US) can suggest CCA. A definitive diagnosis and identification of more subtle associated neurological or nonneurological abnormalities are difficult to be detected by prenatal 2D US; therefore, a further study by fetal magnetic resonance imaging (MRI) is needed. This study highlights the role and evaluates the diagnostic value of fetal MRI in the diagnosis of CCA and identifying the associated anomalies, which is a prognostic of the postnatal developmental outcome.

Results: Out of 27 fetuses that were suspected to have CCA on prenatal US, fetal MRI diagnosed 25/27 fetuses (92.5\%) with CCA, while the last 2/27 (7.5\%) were ruled out; being false positive (FP) on prenatal US, they had normal corpus callosum (CC). Cases diagnosed as CCA were either complete $77.7 \%(n=21)$ or partial agenesis $14.8 \%(n=4)$. They were classified as followed: Only 5/25 (18.5\%) had isolated CCA, while the majority 20/25 (74\%) of CCA were complex form associated with other anomalies (most of them 14 cases (70\%) associated with other neurological anomalies, while 4 cases (20\%) associated with other non-neurological anomalies, and last 2 cases (10\%) were having both anomalies). The diagnostic performance of fetal MRI in diagnosis of CCA was as follows: sensitivity $100 \%$, specificity $67 \%$, positive predictive value (PPV) 96\%, negative predictive value (NPV) 100\%, and accuracy $96.3 \%$. The sensitivity of the detection of associated neurological abnormalities was 100\% by fetal MRI for 75\% by 2D US.

Conclusion: The prevalence of the complex CCA is higher than the isolated form. Fetal MRI is a valuable adjunctive complementary imaging to prenatal 2D US with high diagnostic performance in the diagnosis of fetuses with CCA. It confirms the diagnosis, assesses the extent of agenesis, and detects coexisting anomalies, which is a prognostic of the postnatal developmental outcome.
\end{abstract}

Keywords: Fetal brain, Magnetic resonance imaging, Corpus callosum agenesis

\section{Background}

Corpus callosum (CC) is a major commissures structure in the human brain that consists of white matter tract with millions of myelinated nerve fibers; it connects both cerebral hemispheres. It is composed of four parts: the rostrum, genu, body, and splenium; the isthmus which is the narrow region between the body and splenium [1]. The development of $\mathrm{CC}$ starts between 8 to 11

\footnotetext{
* Correspondence: raniasaberm@hotmail.com

'Diagnostic and Intervention Radiology Department (Pediatric Unit), Faculty

of Medicine, Cairo University, Kasr Al-aini Street, Al-manial District, Cairo, Egypt

${ }^{2}$ Giza, Egypt

Full list of author information is available at the end of the article
}

gestational weeks (GW) in humans, and it is complete in its craniocaudal extent by 18 to $19 \mathrm{GW}$ when it is detected in prenatal imaging [2]. It can be considered fully developed by about 4 years of the child's age. However, further maturation and growth continue throughout life but at a slower rate [3]. Congenital anomalies of CC can be classified as complete agenesis, partial and hypoplasia [4]. The prevalence of CCA is about 0.1 to $0.7 \%$ in the general population and $2.3 \%$ in the developmentally disabled population [2]. The exact etiology is unknown. However, a number of reasons are incriminated including genetic factors; metabolic disorders or developmental insults to the developing brain, including infectious 
diseases [i.e., toxoplasma, rubella, cytomegalovirus, and herpes], or vascular disorders [2].

Isolated CCA is uncommon. In most cases, a prenatally detected CCA is commonly associated with other neurological or non-neurological malformations [2]. The associated neurological malformations are frequently present, and it is the most important prognostic predictors of the postnatal neurological deficits [5]. Routine prenatal 2D US can suggest CCA which relies on detection of indirect signs such as absent cavum septi pellucidi (CSP), ventriculomegaly, widening of the interhemispheric fissure, colpocephaly, high riding 3rd ventricle, and lack of the pericallosal arteries are evident [6]. With the advent of ultrafast T2 imaging techniques, fetal MRI allows better direct visualization of the $\mathrm{CC}$ on a midline sagittal image. Moreover, it allows detection of subtle neurological abnormalities that are prognostic indicators for the postnatal neurodevelopmental outcome [7]. This study emphasizes the diagnostic value of fetal MRI in the detection of CCA and identification of the associated neurological and nonneurological anomalies, which give insight on the postnatal developmental outcome.

\section{Methods}

\section{Patients}

The current study prospectively enrolled 27 pregnant females; 26 cases were singleton pregnancies, and 1 case was dichorionic diamniotic twin pregnancy with 1 fetus suspected to have CCA. They were referred from Fetal Obstetric Ultrasound Unit to Radiology Department with suspected CCA based on prenatal US for further evaluation by fetal MRI. This study has been carried out between January 2018 and December 2019. The maternal ages ranged from 17 to 41 years with a mean \pm SD $(29.5 \pm 5.97$ years) and gestational age (GA) based on last menstrual period; it ranged from 20 to $37 \mathrm{GW}$ with a mean $\pm \mathrm{SD}(31 \mathrm{GW}$ and 4 days $\pm 5.2 \mathrm{GW})$.

\section{Inclusion criteria}

- The affected twin of the dichorionic diamniotic twin pregnancy.

- Incidentally discovered or suspected fetus with CCA monitored by fetal US, especially those fetuses with limited US examination (e.g., maternal obesity, oligohydramnios, fetal position).

- Fetuses with suspected associated congenital anomalies which were not adequately assessed by US.

\section{Exclusion criteria:}

- Fetuses with other central nervous system (CNS) malformations other than CCA.
- Pregnant female in the 1st trimester; (MRI examination is not recommended during organogenesis and due to a small-sized fetus with excessive motion artifact).

- Patients with contraindications for fetal MRI; (e.g., a pacemaker, aneurysm clip, or metallic foreign body)

- Patients who refused the examination.

\section{Imaging}

Patients were advised to fast for $4 \mathrm{~h}$ before the examination to reduce bowel peristalsis artifacts and to prevent postprandial fetal motion. Also, patients were advised to empty the urinary bladder immediately before undergoing fetal MRI to prevent the urinary urge during the examination. All metallic objects were removed. No contrast agents or sedation (maternal or fetal) was used. All fetal MR examinations were performed using two MRI $1.5 \mathrm{~T}$ superconducting scanners (Intera, Philips Medical Systems, Best, Netherlands and Magnetom Aera, Siemens Healthineers, Germany). A multi-channel phased array torso coil was used to allow increase signal to noise ratio, increased coverage of the fetal head, and improve the image quality. Patients were positioned in the supine position (if $<30$ weeks) or in left lateral position (if $>30$ weeks) to allow comfort of the patient during the examination with feet first and ear plugs were offered. The examination time ranged from 20 to 30 min. First maternal scout images were obtained using true fast imaging with steady-state free precession sequence using 6-mm slices in the maternal coronal, axial, and sagittal planes. These images are used to position the subsequent high-resolution sequences adapted to the position of the fetus. This was followed by T1-weighted images (WI) sequence of the brain in axial plane. It was used to detect hemorrhage or fat, sub-ependymal nodules, and calcifications.

Ultra-fast T2-WI single-shot fast spin echo (SSFSE) and balanced fast field of echo (B-FFE) were the mainstay of the examinations and were acquired during maternal breath holding in the axial, sagittal, and coronal planes. Whenever possible, imaging was repeated in each plane until adequate images were obtained. The following imaging parameters were used: $\mathrm{TE}=$ shortest (up to $2 \mathrm{~ms}$ ), TR $=$ shortest (up to $4 \mathrm{~ms}$ ), slice thickness of $2-4$ $\mathrm{mm}$ with a $0-2 \mathrm{~mm}$ gap, large FOV $=300-450 \mathrm{~mm}$, flip angle $=60-90^{\circ}$, number of excitations $=6-18$, and matrix about $1.5-\mathrm{mm}$ in-plane resolution with $18-20$ sequentially acquired imaging slices. All fetal MR images were reviewed by 2 pediatric radiologists (S.E.EL. and R.I. with more than 10 years' experience). Fetal MRI readers were blinded to the exact clinical history and prenatal US results at the time of review. In this study, CC shape, signal intensity, and length were evaluated. Prenatal diagnosis of complete CCA was established in 
the absence of its visualization in mid-sagittal plane, and a diagnosis of partial CCA was diagnosed at numerical values of the length of the $\mathrm{CC}$, less than the 5th percentile of normative indices by Parazzini et al. [8] were considered for pregnancies between 18 and 22 weeks, and those of Garel et al. [9] were utilized for pregnancies between 22 and 37 weeks. Follow-up information about the outcome of the pregnancy, postnatal MRI studies, and autopsy results were reviewed. This study was approved by the hospital research ethics committee. All patients were counseled and signed a consent form.

\section{Statistical analysis}

Data were coded and entered using the Statistical Package for Social Sciences, (SPSS 20) (Inc., Chicago, IL, USA). Data were summarized using range, mean, and standard deviation, in the quantitative data, using frequency (count) and relative frequency (percentage) in the categorical data. Standard diagnostic indices including sensitivity, specificity, positive predictive value (PPV), negative predictive value (NPV) and diagnostic accuracy were calculated.

\section{Results}

A total of 27 pregnant females participated in this study. The maternal age ranged from 17 to 41 years with a mean 29.5 years $(\mathrm{SD}= \pm 5.97)$, and the GA ranged from 20 to $37 \mathrm{WG}$, with a mean $31 \mathrm{WG}$ and 4 days ( $\mathrm{SD} \pm 5.2 \mathrm{WG}$ ). Concerning the maternal obstetric history, the majority 20 cases were multi-gravida (74\%). Regarding their consanguinity, only 9 (33.3\%) had positive consanguinity. Concerning the previous history of fetal CNS anomalies, only 5 (18.5\%) gave history of previous CNS anomalies. Regarding the fetal gender, the majority were males, 19 fetuses (70.3\%); demographic data are presented in Table 1. The fate of pregnancy was 5 cases (18.5\%) underwent termination of pregnancy (abortion), 2 cases (7.5\%) had intrauterine fetal death, while the majority 20 cases (74\%) continued their pregnancy to the 37th WG.

Out of 27 fetuses who were suspected to have CCA on prenatal US, fetal MRI diagnosis agreed with prenatal

Table 1 Demographic details of pregnant females participated in the study

\begin{tabular}{|c|c|}
\hline Number of all participants & $n=27$ \\
\hline Maternal age (mean/+SD) & $\begin{array}{l}29.5 \text { years } \\
(S D= \pm 5.97)\end{array}$ \\
\hline $\mathrm{GA}($ mean/ $\pm \mathrm{SD})$ & $\begin{array}{l}31 \mathrm{GW}, 4 \text { days } \\
(\mathrm{SD} \pm 5.2 \mathrm{GW})\end{array}$ \\
\hline Positive consanguinity & $n=9(33.33 \%)$ \\
\hline Multigravida & $n=20(74 \%)$ \\
\hline Previous offspring with CNS anomalies & $n=5(18.5 \%)$ \\
\hline Fetal male gender & $n=19(70.3 \%)$ \\
\hline
\end{tabular}

US in $25 / 27$ fetuses (92.5\%); they had CCA, while there were disagreements, FP on prenatal US in $2 / 27$ (7.5\%), as they had normal CC by pre- and postnatal MRI. They were classified into either complete $77.7 \%(n=21)$ or partial agenesis $14.8 \%(n=4)$ (Fig. 1). Regarding their association with other anomalies, only $5 / 25(18.5 \%)$ had isolated CCA, while the majority 20/25 (74\%) of CCA cases were complex form associated with other anomalies (most of them 14 cases $(70 \%)$ associated with other neurological anomalies, while only 4 cases $(20 \%)$ associated with other non-neurological anomalies, and the last 2 cases (10\%) had both anomalies) (Fig. 2).

In this study there were $19 / 27$ cases $(70.4 \%)$ that had ventriculomegaly (Fig. 3). Regarding the detection of associated neurological anomalies, the sensitivity of fetal MRI was $100 \%$ compared to $75 \%$ in prenatal US; fetal MRI identified all 16 cases that had other neurological anomalies in the form of; 6/16 cases (37.5\%) had interhemispheric cyst (IHC) (Fig. 4), 5 cases (31.3\%) had neural tube defect (NTD) (Figs. 5, 6, 7, 8, 9, 10, and 11), $5 / 16$ cases $(31.3 \%)$ had cerebellarhemispheric and/ or vermian hypoplasia (Figs. 4 and 6), 3 cases (18.8\%) had tonsillar herniation (Fig. 5), 3 cases (18.8\%) had delayed and abnormal cerebral sulcation (Figs. 4, 5, and; 8), 1 case (6.3\%) had periventricular germinal matrix hemorrhage (GMH) (Fig. 5), 1 case with microcephalic skull $(6.3 \%)$ and 1 case brain stem hypoplasia (6.3\%) (Table 2). anomalies, they were identified equally by prenatal US and MRI in 5 cases. In one case, MRI added additional findings in the diagnosis of associated nonneurological anomalies which were missed by prenatal US. The associated non-neurological findings were renal abnormalities (Fig. 3), thick nuchal fold, skeletal abnormalities (Fig. 2), duodenal atresia with polyhydramnios, and last case with a diaphragmatic hernia illustrated in Table 2.

Upon correlating the fetal MRI findings to the final diagnoses (autopsy or postnatal MRI), 24 cases (88.9\%) were true positive (TP); two cases $(7.5 \%)$ were false positive (FP) by US (7.5\%), being true negative (TN) by MRI. MRI did not miss the diagnosis in any of the cases false negative $(\mathrm{FN}=0)$. While one case $(3.7 \%)$ was FP diagnosed. Based on the previous findings, the diagnostic performance of fetal MRI in diagnosis of CCA was as follows: sensitivity $100 \%$, specificity $67 \%$, PPV 96\%, NPV 100, and accuracy $96.3 \%$ (Fig. 11).

\section{Discussion}

The extent of CC malformation varies from partial to complete agenesis or just hypoplasia; its thickness decreases along all its parts. In partial agenesis, the posterior part is not developed. However, cases of anterior agenesis have also been reported [10]. In CCA, identification of indirect signs of CCA in the axial planes of 


\section{Fetal MRI Corpous Callosum Diagnosis}

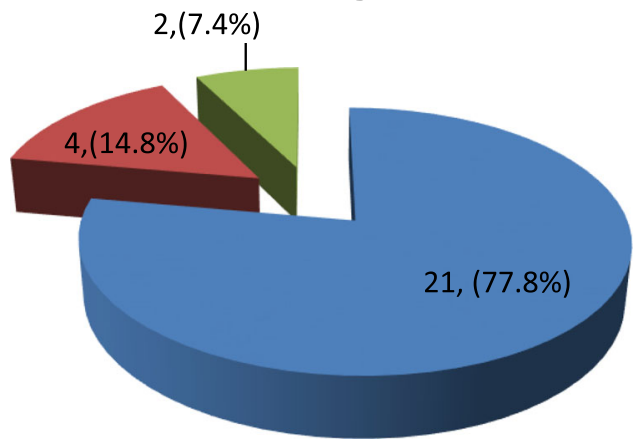

- Complete CCA

- Partial CCA

Normal CC

Fig. 1 Fetal MRI classification of CCA; (partial and complete agenesis)

scanning allows suspecting this pathology. In order to make the final diagnosis, it is necessary to assess $\mathrm{CC}$ in the middle sagittal plane [7]. Fetal MRI allows better direct visualization of the CC. Moreover, the increased contrast resolution of fetal MRI allows detection of subtle CNS abnormalities that are prognostic indicators for the postnatal neurodevelopmental outcome [7]. Many disciplines, including obstetric, perinatology, genetics, pediatric surgery, and neurology, are involved in fetal MRI; therefore, the future of fetal MRI can be achieved through multidisciplinary collaborative team [11].

A total of 27 pregnant females participated in this study according to the inclusion criteria suspected to have CCA on prenatal US. The maternal age ranged from 17 to 41 years with a mean 29.5 years $(S D= \pm 5.97)$. The complete development of CC is around 18 to $19 \mathrm{GW}$ when it is detected in prenatal imaging [2]. Therefore, in this study the GA ranged from 20 to 37 WG; with a mean $31 \mathrm{WG}$ and 4 days (SD \pm 5.2 WG). Concerning the maternal obstetric history, most of the pregnant females, 20 cases, were multi-gravida (74\%), while only 7 cases (26\%) were primi-gravida. Regarding their consanguinity, only 9 (33.3\%) had positive consanguinity, while the majority 18 cases $(66.7 \%)$ were not relatives. Concerning the previous history of fetal CNS anomalies; only 5 (18.5\%) gave history of previous CNS anomalies in their offsprings. Regarding the fetal gender, the majority were males, 19 fetuses $(70.3 \%)$ while 8 fetuses $(29.7 \%)$ were females; these were coinciding with a study conducted by Manganaro et al. [12]: they revealed that the average maternal age was 32.7 years (SD $= \pm 5.8$ ), male fetuses were more numerous than female fetuses. Considering the fate of pregnancy, Manganaro et al. [12] found that $27 \%$ were terminated; $5.8 \%$ were stillbirths; and $22 \%$ were born. In the current study, 5 cases $(18.5 \%)$ underwent termination of pregnancy

\section{Fetal MRI Corpous Callosum Diagnosis.}

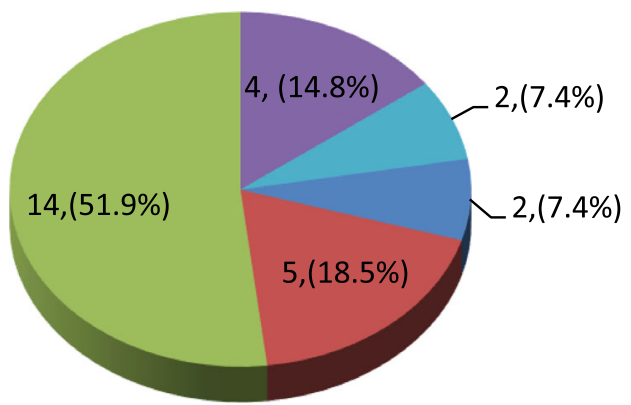

Normal CC

- Isolated CCA

Associated neurological anomalies

Associated nonneurological anomalies

Fig. 2 Descriptive statistics of fetal MRI of CCA; (normal CC, isolated, and non-isolated CCA) 

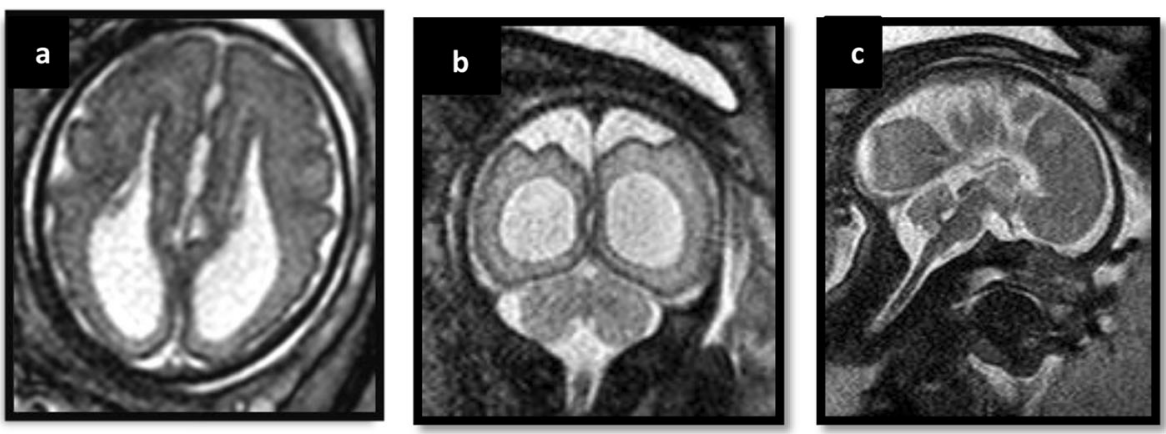

Fig. 3 A 29-year old pregnant female, 30th WG, (G2P1L1). T2 HASTE MRI of fetal brain in axial (a), coronal (b) views show: Parallel orientation of the bodies of both lateral ventricles, being widely separated laterally with dilated posterior horn (colpocephaly). c Mid-sagital view shows the medial sulci and the adjacent cortical gyri are radiating in "sun burst" pattern perpendicular to the expected course of the corpus callosum and absent pericallosal sulcus. Diagnosis: Picture suggestive of isolated complete CCA

(abortion), 2 cases $(7.5 \%)$ had intra-uterine fetal death, while the majority 20 cases $(74 \%)$ continued their pregnancy to 37th WG.

Out of 27 fetuses who were suspected to have CCA on prenatal US, fetal MRI diagnosis agreed with prenatal US in 25/27 fetuses (92.5\%); they had CCA, while there were disagreements in $2 / 27$ (7.5\%) FP on prenatal US; they had normal CC by pre- and postnatal MRI. A study conducted by Sakar et al. [13] suspected 21\% CC abnormality by US. MRI confirmed in about $83 \%$ of cases while in rest (16.7\%), it disproved US diagnosis.

In the current study, CCA were classified into either complete $77.7 \%(n=21)$ or partial agenesis $14.8 \%(n=$ 4 ), and no hypoplastic cases. This was in accordance
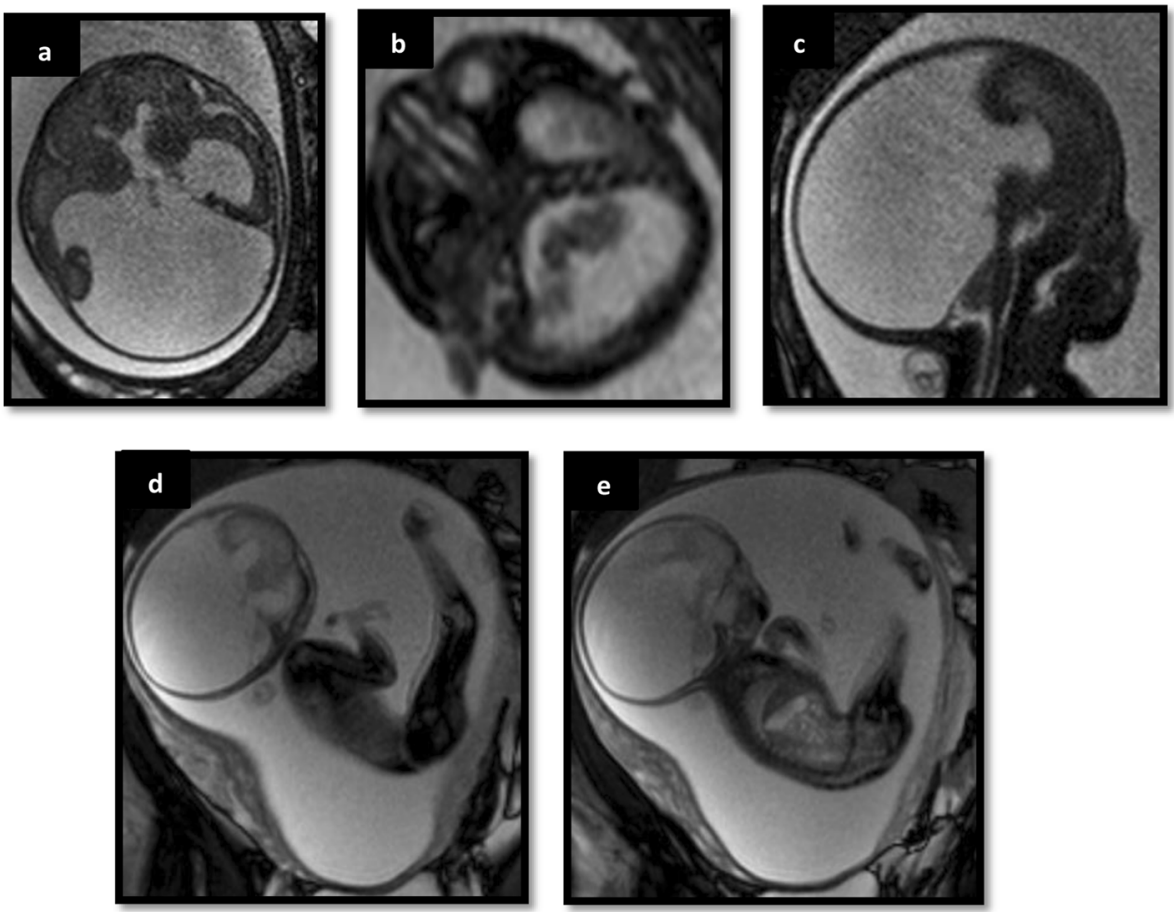

Fig. 4 A 20-year old pregnant female at 30th WG, G1POLO, and negative consanguinity. T2* B-FFE-weighted MR images of the fetal brain $\mathbf{a}$ and $\mathbf{b}$ Axial and $\mathbf{c}$ sagittal views: shows typical indirect signs of CCA; supratentorial hydrocephalic changes, with colpocephaly, deformed and upwards vertically oriented frontal horns and upwards extension of dilated 3rd ventricle, large dorsal inter-hemispheric cyst, also diffusely abnormal delayed sulcation pattern. Dilated retrocerebellar space with small cerebellar hemispheres and brain stem, absent inferior vermis. $\mathbf{d}$ and e sagittal view of the whole fetal body shows polyhydramnios, bilateral clenched hands, bilateral talipes. Diagnosis: Partial CCA, inter-hemispheric cyst, lissencephaly, cerebellar and brain stem hypoplasia, polyhydraminos bilateral talipes, and bilateral clenched hands 

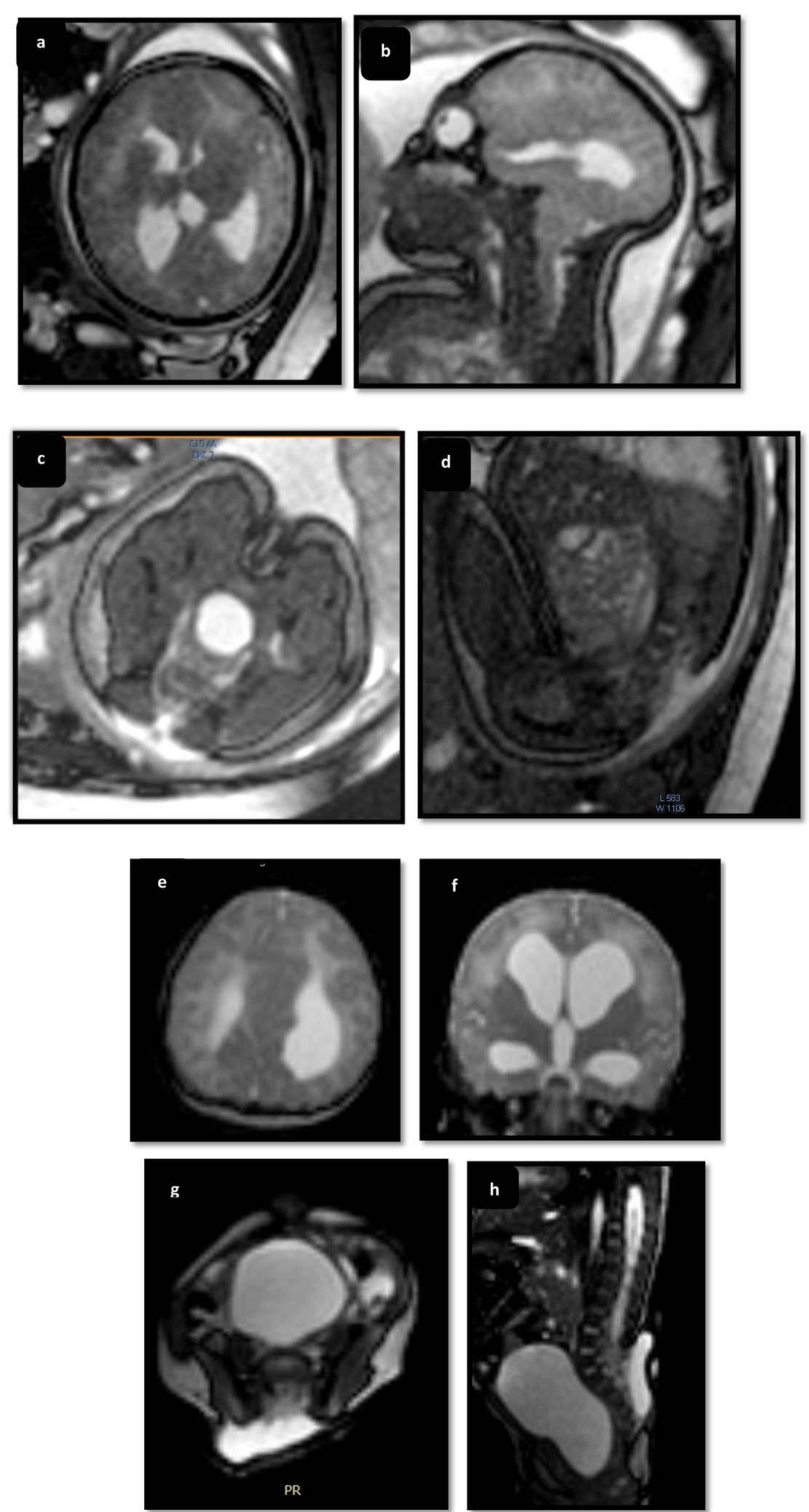

Fig. 5 (See legend on next page.) 
(See figure on previous page.)

Fig. 5 A 29-year old pregnant female at 37th WG, with obstetric history of giving birth to a normal child, one previous abortion, and positive consanguinity. T2*B-FFE FMRI (a and $\mathbf{b}$ ) axial and sagittal images of the brain show supratentorial hydrocephalus, colpocephaly, absent corpus callosum, sulcation delay (arrows), crowded posterior fossa, and tonsillar herniation (c and $\mathbf{d}$ ) axial and sagittal images of the spine show lumbosacral meningocele (arrows). Postnatal BFFE MRI brain (e) and (f) and spine (g) and (h) confirm the diagnosis. Diagnosis: Complete CCA associated with Chiari II malformation

with Rüland et al. [14]; they diagnosed complete agenesis in $76 \%$, partial agenesis $14 \%$, and a hypoplastic CC in $9 \%$ of cases.

The classification of CCA can be either an isolated anomaly or associated with other abnormalities: neurological or non- neurological anomalies. This study observed that only $5 / 27(18.5 \%)$ had isolated CCA, while the majority 20/27 (74\%) were complex form and associated with other anomalies. This was concordance with a study done by Manganaro et al. [12]; they revealed that fetal MRI demonstrated isolated CCA in $26.9 \%$, while the majority of the cohort about $73.1 \%$ CCA was associated with other anomalies. Another study done by Rüland et al. [14] that found about $29 \%$ was isolated and $71 \%$ was non-isolated form. However, these results are higher than what had been reported in a previous study done by Tang et al. [15]; they found that only $7 \%$ of cases of CCA were isolated by fetal MRI; this may be due to the younger GA and increased number of fetuses in their study.

Ventriculomegaly was not considered an additional finding because dilatation of the atria of the lateral
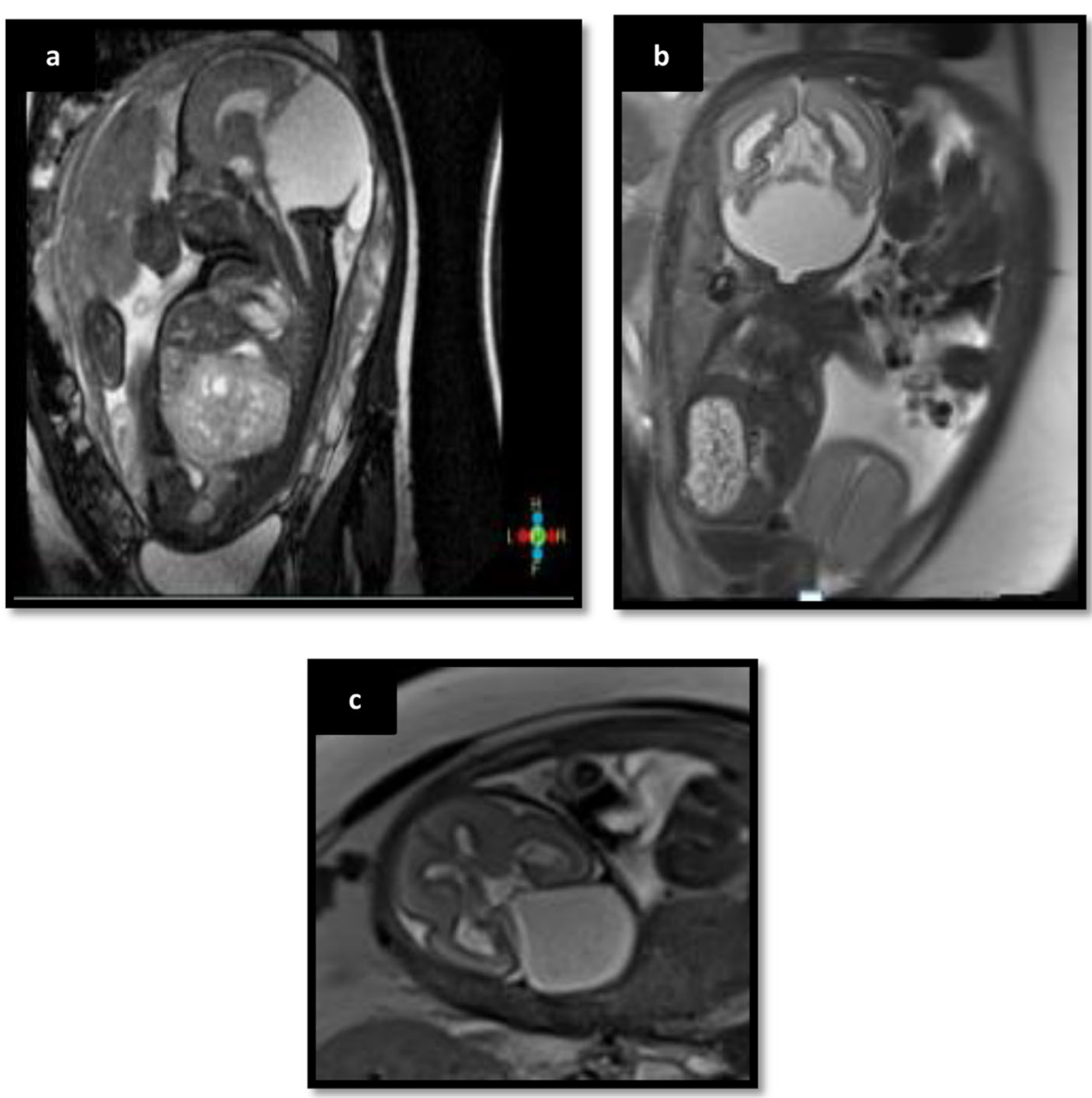

Fig. 6 A 35-year old pregnant female, 25th WG of twin (G2P1L1). MRI of the fetal brain of one twin: a Sagittal, b coronal views of the fetus, and c axial view of fetal brain revealed enlarged posterior fossa showing large cyst which is communicating with the 4th ventricle, as well as occipital meningeocele, hypoplastic laterally displaced cerebellar hemispheres with absent cerebellar vermis. Hypoplastic splenium of the corpus callosum, widely separated occipital horns of the lateral ventricles. Bilateral small eye globes, bilateral enlarged high signal with innumerable small cystic kidneys compressing the liver and shifting of the diaphragm upwards with hypoplastic lung. Diagnosis: Partial CCA associated with Dandy-Walker syndrome, Meckel-Gruber syndrome, bilateral small eye globes, and hypoplastic lungs 

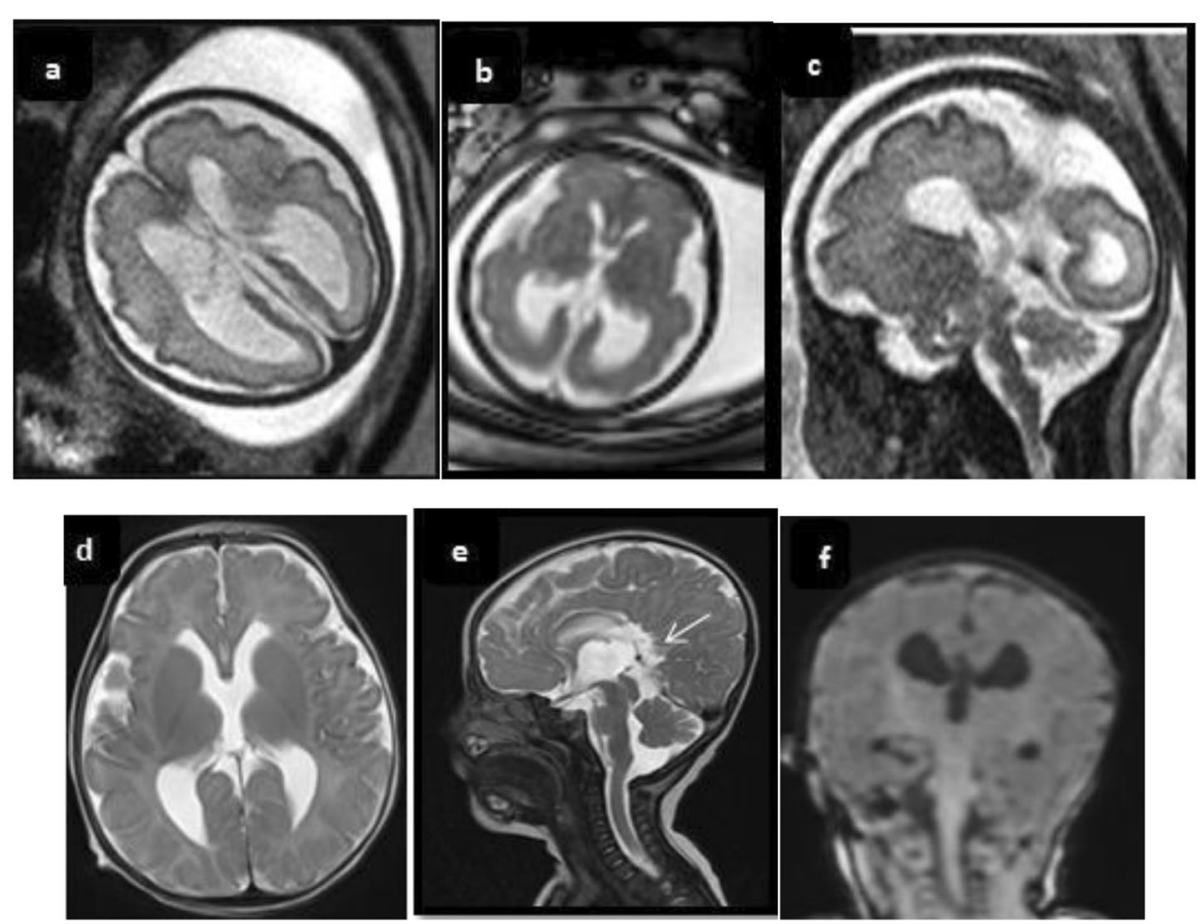

Fig. 7 A 30-year old pregnant female 30 WG, (G4P3L3), with positive consanguinity. MRI of fetal brain axial (a and b), sagittal (c) views show a small anterior part of CC that is lacking its body and splenium and only partially surrounds the 3rd ventricle, mild supratentorial ventriculomegaly with widely separated parallel medial wall of lateral ventricles, and colpocephaly. Post-natal MRI fetal brain follow-up confirms the diagnosis; axial (d) and mid-sagittal (e), coronal (f) views reveal absent CSP, high riding 3rd ventricle, mild supratentorial hydrocephalus, colpocephaly, hypoplasia of the posterior part of the body and splenium of the corpus callosum, the adjacent cortical gyri are radiating in "sun burst" pattern perpendicular to the dilated upward extended 3rd ventricle (arrow), the ventricles taking the appearance of moose head. Diagnosis: Isolated partial agenesis of the corpus callosum
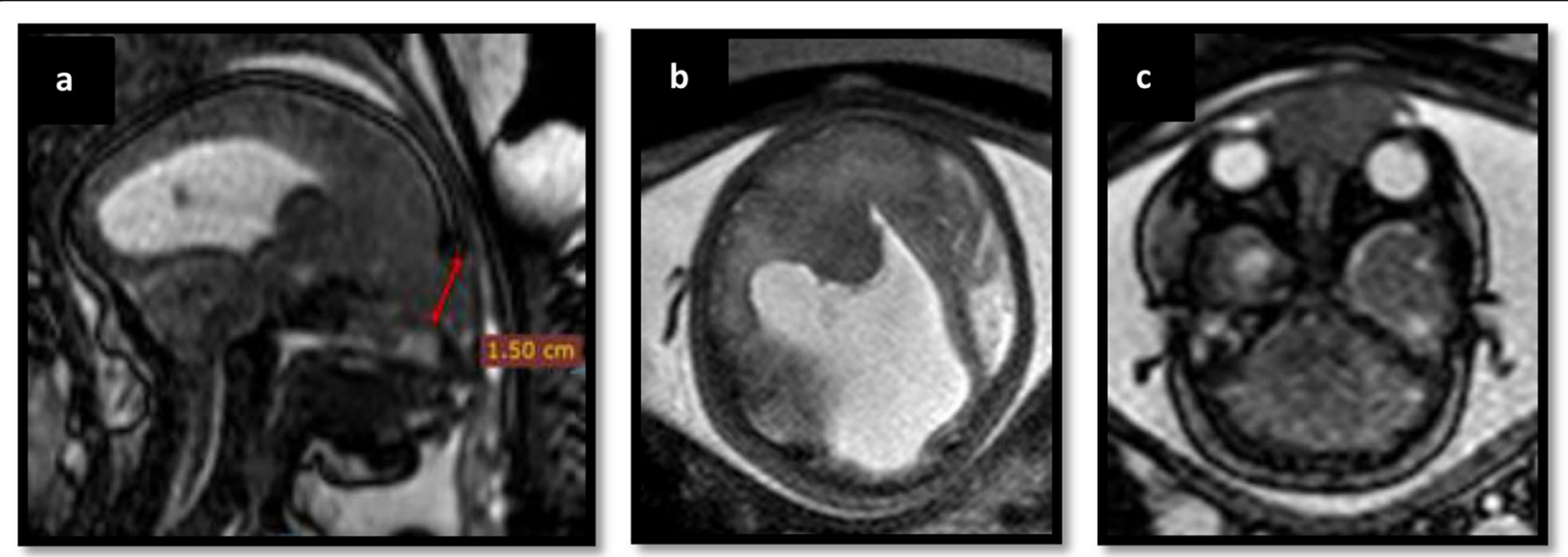

Fig. 8 A 29-year old pregnant female, 35th WG, (G3P2L2). MRI of the fetal brain in sagittal (a) and axial (b and c) views show: Herniation of brain parenchyma through a frontal midline defect measuring about $1.5 \mathrm{~cm}$, associated with mild hypertolerism. Asymmetry of cerebral hemispheres, enlarged right cerebral hemisphere shows abnormal cortical infoldings and sulcation, large right posterior porencephalic cyst is communicating with deformed dilated right lateral ventricle body and the corpus callosum could not be identified, crowded posterior fossa with tonsillar herniation, and small skull size. Diagnosis: CCA, Chiari I with frontal cephalocele, right hemimegalencephaly, right porencephaly, Abnormal sulcal pattern, and microcephalic skull 

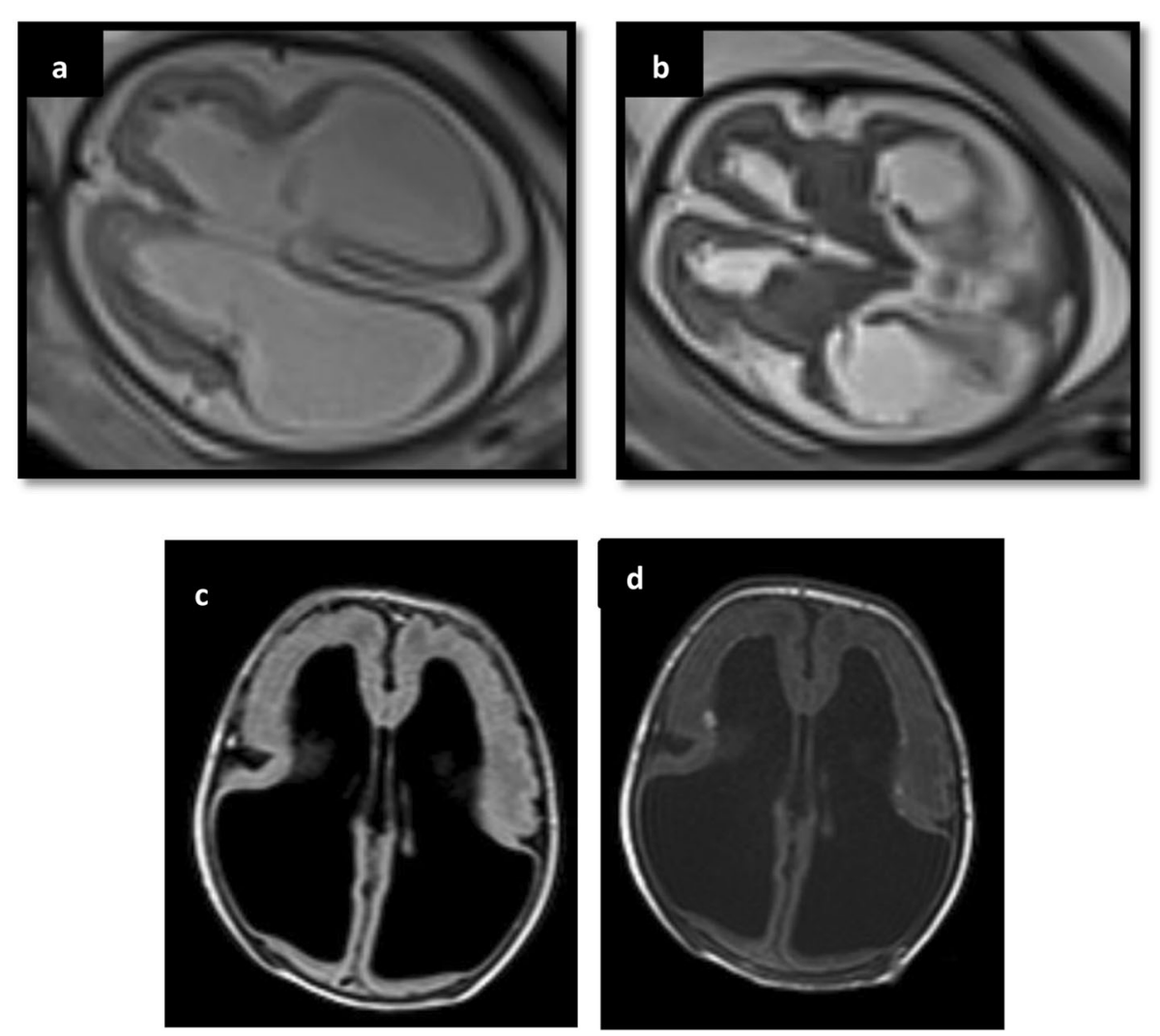

Fig. 9 A 36-years old pregnant female, 30 WG, (G2P1L1). Fetal BFFE MRI of the brain (a and b) T2 WI axial views reveal marked ventriculomegaly of both lateral ventricles with colpocephaly, frontal horns pointed anteriorly, 3rd ventricle upward elevation, thinning of cortical mantle at both posterior parietal regions, bilateral periventricular nodular hypodensity, suspected germinal matrix hemorrhage, delayed sulcal pattern. Postnatal MRI brain (c) FLAIR and (d) T1 WI confirm the diagnosis show punctate periventricular nodular being hypodense in FLAIR image and hyperdense in T1WI (early sub-acute hemorrhage). Diagnosis: CCA with punctate periventricular GMH
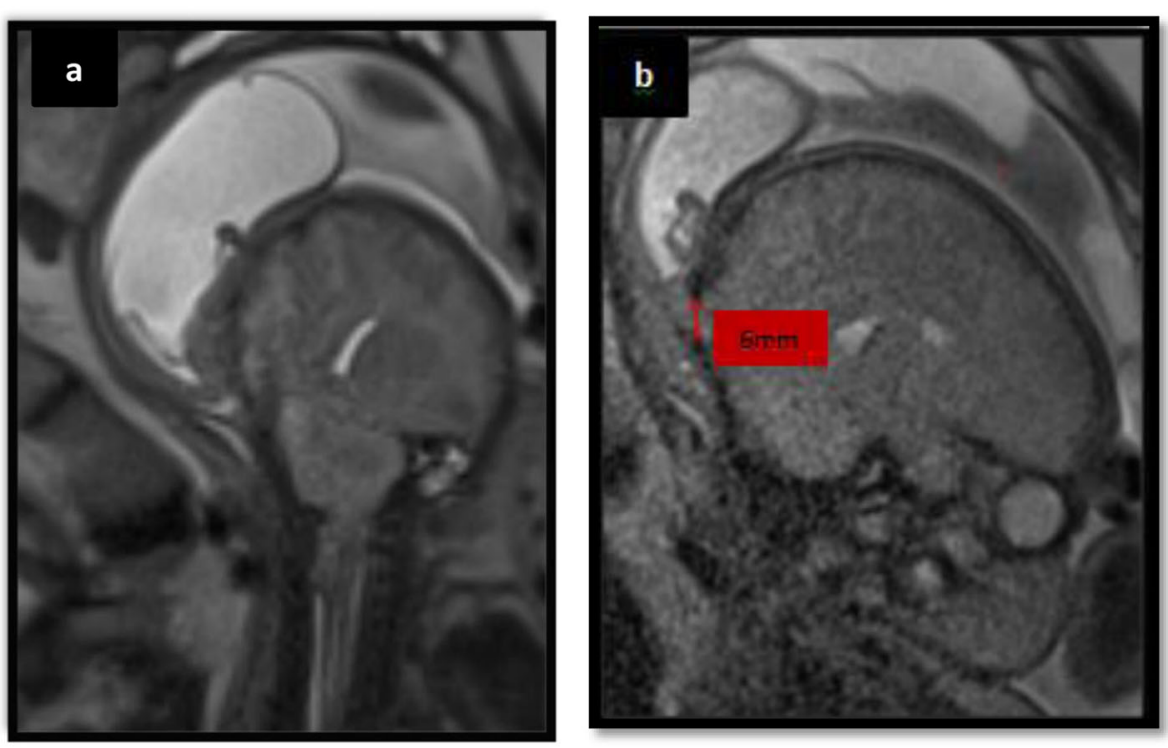

Fig. 10 A 30-year old pregnant female, 34th WG, (G1POLO). Fetal MRI of the brain sagittal T2 WI shows complete absence of the CC with herniation of the brain parenchyma and meninges through occipital defect about $6 \mathrm{~mm}$. Diagnosis: CCA with occipital encephalo-meningeocele 
Table 2 Fetal MRI distribution of neurological and nonneurological findings in CCA cases associated with other anomalies

\begin{tabular}{llll}
\hline $\begin{array}{l}\text { Neurological } \\
\text { anomalies }\end{array}$ & $\begin{array}{l}\text { No. of } \\
\text { cases }\end{array}$ & $\begin{array}{l}\text { Non-neurological } \\
\text { anomalies }\end{array}$ & $\begin{array}{l}\text { No. of } \\
\text { cases }\end{array}$ \\
\hline IHC & $\mathbf{6}$ & $\begin{array}{l}\text { Renal abnormalities } \\
\text { Diaphragmatic hernia }\end{array}$ & $\mathbf{2}$ \\
$\begin{array}{l}\text { Cerebellar/vermian } \\
\text { hypoplasia }\end{array}$ & $\mathbf{5}$ & $\mathbf{1}$ & Duodenal atresia \\
$\begin{array}{l}\text { Neural tube defect } \\
\text { Tonsillar herniation }\end{array}$ & $\mathbf{3}$ & $\begin{array}{l}\text { Hypoplastic lungs } \\
\text { Small eye globe }\end{array}$ & $\mathbf{1}$ \\
$\begin{array}{l}\text { Abnormal cerebral } \\
\text { gyration }\end{array}$ & $\mathbf{3}$ & $\mathbf{1}$ \\
Porencephalic cyst & $\mathbf{1}$ & $\begin{array}{l}\text { Thick nuchal fold (Down } \\
\text { syndrome) }\end{array}$ & $\mathbf{1}$ \\
Microcephalic skull & $\mathbf{1}$ & Skeletal abnormalities & $\mathbf{1}$ \\
Periventricular GMH & $\mathbf{1}$ & & \\
Brain stem hypoplasia & $\mathbf{1}$ & & \\
\hline
\end{tabular}

ventricle (more than $10 \mathrm{~mm}$ ) can be attributed to callosal agenesis; it is the main feature of isolated CCA. In this study, there were $19 / 27$ cases $(70.4 \%)$ that had ventriculomegaly; one of them shows abnormal morphology of the lateral ventricle involving the posterior horn, which is incompletely formed, being deformed in shape, with undulations along its margin, and focally dilated due to communicating with porencephalic cyst.

For cases diagnosed to have complex form of CCA, they are classified into: most of them 14 cases (70\%) associated with other neurological anomalies, while only 4 cases (20\%) associated with other non-neurological anomalies, and the last 2 cases (10\%) had both anomalies.

The most common associated neurological finding was IHC about 6/16 cases (37.5\%), 5 cases (31.3\%) had NTD, $5 / 16$ cases $(31.3 \%)$ of cerebellar hemispheres and vermian hypoplasia, 3 cases (18.8\%) had tonsillar herniation, 3 cases $(18.8 \%)$ had delayed and abnormal cerebral sulcation, 1 case $(6.3 \%)$ had periventricular GMH, 1 case with microcephalic skull (6.3\%), and 1 case brain stem hypoplasia (6.3\%). The sensitivity of fetal MRI compared to prenatal US in detection of neurological abnormalities was $100 \%$, while 2D US was $75 \%$. In a study conducted by Tang et al. [15], fetal MRI identified abnormalities not detected by prenatal sonography in most $(83 \%)$ patients; this difference is higher than that detected with current study in which fetal MRI findings coincided with that observed by prenatal US in 12 cases (75\%). However, MRI added additional information to the US findings in 4 cases only (25\%) in the form of: 1 case with small dorsal IHC, vermian hypoplasia, and small occipital meningocele; 2 cases with tonsillar descent (1 case was Chiari I associated with frontal encephalocele, abnormal sulcation, and hemimegalencephaly), the other case with small sacral meningocele (Chiari II).
A study done by Manganaro et al. [12] found that $39.5 \%$ of cases had cortical developmental defects by fetal MRI. In another study conducted by Tang et al. [15], they observed that $42.8 \%$ of cases had abnormal sulcal morphology, with gyral malformations. However, in the current study, only 3 cases $(18.8 \%)$ had delayed and abnormal cerebral sulcation. Therefore, more sensitive methods such as 3D morphometric study, diffusionweighted, and diffusion tensor imaging might detect other sulcal abnormality cases.

Posterior fossa abnormalities were also a common additional finding, in the form of $5 / 16$ cases $(31.3 \%)$ of cerebellar and/or vermian hypoplasia, 3 cases $(18.8 \%)$ of tonsillar herniation, and 2 cases (12.5\%) of occipital meningocele/encephalocele. This was in agreement with a study done by Manganaro et al. [12]; they confirmed that fetal MRI detected associated cerebellar malformations in 30\%. They also revealed that fetal MRIdiagnosed CCA with cerebellar hemispheric abnormalities were more common than vermian abnormalities; they stated that it may be due to the difficulty in evaluating the fetal vermis or due to termination of those cases with prenatally diagnosed cerebellar abnormalities. However, in current study, fetal MRI diagnosed CCA with both cerebellar hemispheric abnormalities and vermian abnormalities equally.

In the current study, the most common finding was IHC, 6/16 cases (37.5\%), while the least association was periventricular GMH and a microcephalic skull each represented in 1 case only (6.3\%). In a study conducted by Manganaro et al. [12], they detected that about $19.7 \%$ had IHCs and only $9.2 \%$ had microcephaly.

Regarding the associated non-neurological anomalies, Manganaro et al. [12] found that $22.4 \%$ had thoracoabdominal, $18.4 \%$ had musculoskeletal, $17 \%$ had craniofacial, and $14.5 \%$ had cardiac malformations. However, in this study, they were identified equally by prenatal US and MRI in 5 cases. In the last case that had both neurological and non-neurological anomalies, MRI added additional findings in the diagnosis of associated nonneurological anomalies (hypoplastic lung and bilateral small eye globes) which were missed by prenatal US. The most common non-neurological anomalies were renal abnormalities represented in 2 cases; one case in the form of renal pelvic dilatation while the other was bilateral enlarged multicystic kidneys (Meckel-Gruber syndrome), it also had combined anomalies/associated neurological anomalies, which were enlarged posterior fossa showing large cyst which is communicating with the 4th ventricle, as well as small occipital meningocele, hypoplastic laterally displaced cerebellar hemispheres with absent cerebellar vermis, widely separated occipital horns of the lateral ventricles, 1 case with thick nuchal fold (Down syndrome), 1 case of a duodenal atresia with 
subsequent polyhydramnios, 1 case of a diaphragmatic hernia, and last case with skeletal abnormalities (bilateral talipes equiovarus and clenched hands). It also had combined anomalies/associated neurological anomalies in the form of large dorsal IHC, also diffusely delayed sulcation pattern, enlarged retrocerebellar space with small cerebellar hemispheres, absent inferior vermis, and hypoplastic brain stem.

Prenatal detection of CCA presents a challenge as the US signs may be very subtle or appear late after the second-trimester scans [15]. Many studies, such as Wagenvoort et al. [16] and Malinger et al. [17] concluded that MRI is superior to US, particularly in the diagnosis of callosal anomalies. Raafat et al. [18] studied the prevalence and value of fetal MRI in the diagnosis of midline cerebral anomalies. They revealed that 5 cases of partial callosal agenesis were missed by US and diagnosed by MRI. The diagnostic accuracy for detecting failed commissuration was $86.49 \%$ for US and $100 \%$ for MRI. In the current study, upon correlating the fetal MRI findings to the final diagnoses, 24 cases (88.9\%) were true positive (TP) and two cases (7.5\%) were false positive (FP) by US (7.5\%), being true negative (TN) by MRI as they had a normal CC. MRI did not miss the diagnosis in any of the cases false negative $(\mathrm{FN}=0)$. While one case (3.7\%) was FP diagnosed as isolated partial CCA, which was having a normal CC in postnatal MRI. The diagnostic performance of fetal MRI in diagnosis of CCA was as follows: sensitivity $100 \%$, specificity $67 \%$, PPV 96\%, NPV 100, and accuracy $96.3 \%$ to accuracy of $88.8 \%$ for prenatal US.

Superior soft tissue contrast, relative operator independence, and a large field of view of fetal MRI make it a valuable adjunctive complementary imaging to prenatal 2D US with high diagnostic performance in fetuses with CCA. However, MRI still has some disadvantages which include fetal motion artifact which affects image quality and its ability to obtain a true sagittal plane, although it is reduced significantly by using the ultrafast MR techniques; poorer spatial resolution than US; high cost; and lack of expertise. MRI showed superior value to US in diagnosis of IHC, vermian abnormalities, tonsillar herniation, NTD, and abnormal cerebral sulcation. While in nonneurological anomalies MRI was superior in detection of hypoplastic lung and small eye globes. The result is not a technical advantage of the imaging method but due to the fact that fetal MRI examination is always done for a specific organ of concern not for whole fetal body; however, prenatal US is used for screening the whole body for fetal anomalies.

This study highlights the diagnostic value of the fetal MRI in the diagnosis of CCA; it has the ability to prognosticate the developmental outcome through detection of associated anomalies and allows informing the family about the neurodevelopmental outcome of the child. Therefore, continuous extended broad researches of fetal MRI of CCA should be done, and longer follow-up is needed because behavioral and cognitive difficulties may not be detected until school age.

The study is limited by the fact that it included only fetuses with prenatal US diagnosis of CCA and excluded the cases with other brain anomalies; also, the study relied on the prenatal US diagnosis; which depend on the judgment of obstetrician whose learning curve was probably variable as US is an operator-dependent study. Furthermore, the readers were from the same institution; therefore, some variability in results if other institutions replicated the work cannot be ruled out. Finally, fetal MRI was performed at many different GA, which is likely affecting the sensitivity of fetal MRI for CCA diagnosis; thus, performing fetal MRI at a consistent GA would probably give a better idea of the accuracy of the study.

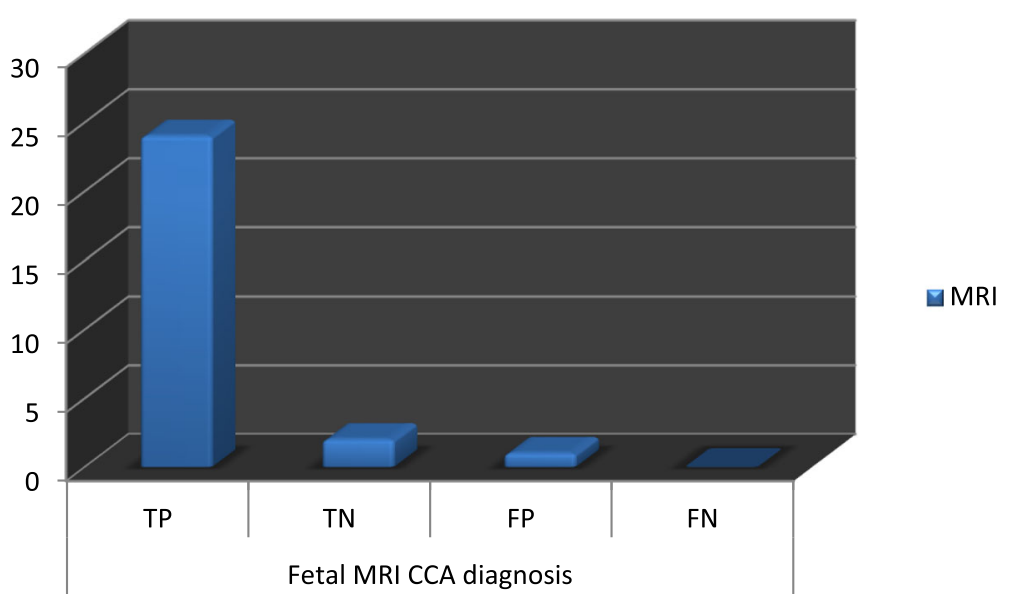

Fig. 11 Statistical measures of fetal MRI performance in diagnosis of CCA and associated anomalies 


\section{Conclusion}

The prevalence of the complex CCA is higher than the isolated form. US is the main primary survey; however, MRI can add more additional information; it confirms the diagnosis, assesses the extent of agenesis, and detects coexisting anomalies; which is a prognostic of the postnatal developmental outcome.

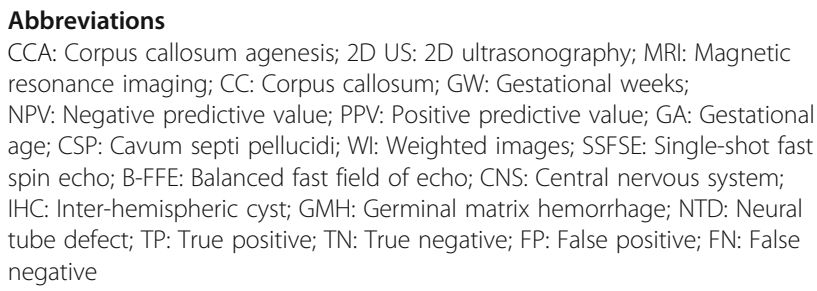

\section{Acknowledgements}

The authors wish to acknowledge the assistance of Obstetric and pediatric departments' staff, for their contribution to patient data collection.

\author{
Author contributions \\ All authors have read and approved the manuscript. \\ - Study concept: S.E.E \\ - Study design: R.I., S.E.E. \\ - Data acquisition: R.I. \\ - Data analysis and interpretation: S.E.E., R.I \\ - Statistical analysis: R.I. \\ - Manuscript preparation: R.I \\ - Manuscript editing: R.I. \\ - Manuscript reviewing: R.I, S.E.E.
}

\section{Funding}

Self-funding.

\section{Availability of data and materials}

The datasets used and/or analyzed during the current study are available from the corresponding author on reasonable request.

\section{Ethics approval and consent to participate}

This study was approved by Radiology Department Kasr Alaini Cairo University hospital research ethics committee. Number: not available. Written informed consent was signed by patients.

\section{Consent for publication}

All patients included in this research gave written informed consent to publish the data contained within this study.

\section{Competing interests}

"The authors declare that they have no competing interests."

\section{Author details}

${ }^{1}$ Diagnostic and Intervention Radiology Department (Pediatric Unit), Faculty of Medicine, Cairo University, Kasr Al-aini Street, Al-manial District, Cairo, Egypt. ${ }^{2}$ Giza, Egypt. ${ }^{3}$ Diagnostic and Intervention Radiology Department (Pediatric Unit), Cairo University Hospitals, Kasr Al-aini Street, Al-manial District, Cairo, Egypt.

Received: 19 February 2020 Accepted: 15 April 2020

Published online: 08 May 2020

\section{References:}

1. Raybaud CH (2010) The corpus callosum, the other great forebrain commissures, and the septum pellucidum: anatomy, development, and malformation. Neuroradiology 52(6):447-477

2. Glenn OA, Goldstein RB, Li KC, Young SJ, Norton ME, Busse RF, Goldberg JD et al (2005) Fetal magnetic resonance imaging in the evaluation of fetuses referred for sonographically suspected abnormalities of the corpus callosum. J Ultrasound Med. 24(6):791-804

3. Tanaka-Arakawa MM, Matsui M, Noguchi K (2015) Developmental changes in the corpus callosum from infancy to early adulthood: a structural magnetic resonance imaging study. Public Liberary of Science 10(7):1-13

4. Kazi IYAl, Kazi SE (2015) Antenatal diagnosis of corpus callosal agenesis. J. Fetal Med. 2:87-90

5. Moutard M. L., Kieffer V., Feingold J., Billette TH., Lewin F., Baron J.M. (2012) Isolated corpus callosum agenesis: a ten-year follow-up after prenatal diagnosis (How are the children without corpus callosum at 10 years of age?. Prenatal Diagnosis 32(3):277-283.

6. Paladini D, Pastore G, Cavallaro A, Massaro M, Nappi C (2013) Agenesis of the fetal corpus callosum: sonographic signs change with advancing gestational age. Ultrasound Obstet Gynecol 42:687-690

7. Glenn OA (2010) MR imaging of the fetal brain. Pediatr Radiol 40:68-81

8. Parazzini C, Righini A, Rustico M, Consonni D, Triulzi F (2008) Prenatal magnetic resonance imaging: brain normal linear biometric values below 24 gestational weeks. Neuroradiology 50(10):877-883

9. Garel C (2004) MRI of the fetal brain: normal development and cerebral pathologies, pathology of the midline cap. Springer, Berlin:131-149

10. Radhouane A, Khaled N (2016) Corpus callosum agenesis: role of fetal magnetic resonance imaging. Asian Pacific Journal of Reproduction 5(3): 263-265

11. Da Silva NA, Vassallo J, Sarian LO, Cognard CH, Sevely A (2018) Magnetic resonance imaging of the fetal brain at 3 Tesla. Preliminary experience from a single series. Medicine 97(40):1-6

12. Manganaro L, Bernardo S, De Vito C, Antonelli A, Marchionni E, Vinci V et al (2017) Role of fetal MRI in the evaluation of isolated and non-isolated corpus callosum dysgenesis: results of a cross-sectional study. Prenatal Diagnosis 37:244-252

13. Sarkar P (2017) Fetal magnetic resonance imaging: a problem solving tool in antenatally detected fetal anomalies and abnormalities. Int J Sci Study. 4(10):142-147

14. Rüland AM, Berg C, Gembruch U, Geipel A (2016) Prenatal diagnosis of anomalies of the corpus callosum over a 13-year period. Ultraschall Med. 37(6):598-603

15. Tang Al, Bartha ME, Norton AJ, Barkovich EH, Glenn OA (2009) Agenesis of the corpus callosum: an MR imaging analysis of associated abnormalities in the fetus. Am J Neuroradiol 30:257-263

16. Wagenvoort AM, Bekker MN, Go AT, Vandenbussche FB, van Buchem VJ, van Vugt JM (2000) Ultrafast scan magnetic resonance in prenatal diagnosis. Fetal Diagn Ther 15:364-372

17. Malinger G, Ben-Sira L, Lev D, Ben-Aroya Z, Kidron D, Lerman-Sagie T (2004) Fetal brain imaging: a comparison between magnetic resonance imaging and dedicated neurosonography. Ultrasound Obstet Gynecol 23:333-340

18. Raafat MER, Tamer M, Abdelrahman TM, Hafez MAF (2020) The prevalence and the adding value of fetal MRI imaging in midline cerebral anomalies. Egyp J Radiol Nuclear Med 51(31):1-8

\section{Publisher's Note}

Springer Nature remains neutral with regard to jurisdictional claims in published maps and institutional affiliations.

\section{Submit your manuscript to a SpringerOpen ${ }^{\circ}$ journal and benefit from:}

- Convenient online submission

- Rigorous peer review

- Open access: articles freely available online

High visibility within the field

- Retaining the copyright to your article

Submit your next manuscript at $\boldsymbol{\sim}$ springeropen.com 